\title{
La selección del tren motriz basada en la eficiencia energética para vehículos de servicio pesado
}

\author{
M.Y. Rafael-Morales y J. Cervantes de Gortari \\ Instituto Mexicano del Transporte y Departamento de Termoenergía \\ Facultad de Ingeniería, UNAM \\ E-mails:mrafael@imt.mxy jgonzalo@servidor.unam.mx
}

(recibido: marzo de 2001; aceptado: junio de 2003)

\begin{abstract}
Resumen
Entre los problemas que afectan a la economía del sector de autotransporte en México, especialmente en el transporte destinado al servicio pesado, se encuentra el de baja eficiencia en el consumo de combustible. No obstante, la avanzada tecnología que generalmente se va disponiendo y adoptando en el parque vehicular con relación a la eficiencia energética, el costo por tonelada transportada no ha disminuido de manera importante. En este trabajo, se presenta un estudio acerca de la influencia que tiene dentro de esta problemática la selección de los componentes del tren motriz en el consumo de combus tible y en la capacidad de ascenso del vehículo, en relación con el peso de la carga transportada. Como resultado del estudio se desarrolló un algoritmo basado en las pruebas reales de desempeño a que puede someterse un vehículo, buscando el régimen óptimo de economía del combus tible. El programa permite a las empresas de transporte, seleccionar el tren motriz más adecuado para cada vehículo teniendo en cuenta las operaciones a que deberá sujetarse en un determinado servicio.
\end{abstract}

Descriptores: autotransporte pesado, tren motriz, tracción, ahorro energético.

\begin{abstract}
Trans porta tion activities in Mexico, es pe cially in heavy-duty ve hicles, are affected by the low ef ficiency in fuel con sump tion. In spite of given the ad vanced tech nol ogy in en ergy eficiency that gen er ally is adopted and used in the Mex i can cargo fleet, there is not a sig nif $f$ cant re duc tion in the op er a tion costs of this very im portant eco nomic ac tiv ity. In this paper, an account about the in flu ence that the se lection of the traction sys tem components has on the fuel con sump tion and the as cend ing ca pac ity of the ve hicle is pre sented. As a re sult of the study, an al go rithm based on real per for mance tests with op ti mal fuel econ omy, was de veloped. The pro gram is use fulfor the se lection of the most ap pro priate traction syst em of a given ve hi cle, tak ing into ac count the con di tion that has to ful fill for a cer tain task.
\end{abstract}

Keywords: heavy-duty ve hicles, trac tion, fuel con sump tion. 


\section{Introducción}

El transporte pesado en México utiliza vehículos que han sido seleccionados de manera tradicional, esto es, basándose en la experiencia de los operadores, en preferencias personales de los empresarios transportistas o en recomendaciones comerciales (CCE,1993). Esto ha provocado una deficiente operación de los mismos, debido principalmente a la falta de conocimiento que existe respecto al funcionamiento de los componentes del tren motriz en el desempeño de la unidad y de su relación con el consumo de combustible, así como con el peso de la carga transportada. A manera de ejemplo se puede señalar que durante el inicio de las operaciones del servicio de transporte de lujo para pasajeros, las primeras unidades importadas en México, presentaron fallas en el tren motriz durante su operación, debido a que fue seleccionado para condiciones geográficas diferentes a las de nuestro país.

Una selección adecuada de los elementos que integran el tren motriz de la unidad favorece la correcta operación de las unidades de acuerdo con las características del servicio que presten. Es necesario entonces, que el transportista cuente con elementos que le ayuden a seleccionar y analizar técnicamente el desempeño del tren motriz de los vehículos que tenga que adquirir, ya sea por renovación o por reposición de las unidades (Berg,1996).

El procedimiento de selección del tren motriz no es una tarea fácil de realizar, ya que cada mecanismo que forma parte del tren se encuentra relacionado con los demás componentes, por lo que cualquier modificación que se realice en alguno de ellos afecta al desempeño del tren motriz en su conjunto. Dicha selección debe cumplir además con la reglamentación vigente para la operación de los vehículos. Esto hace que la selección resulte un proceso que requiere gran consumo de tiempo para su realización, por lo que se estudió la necesidad de contar con un procedimiento sistématico de selección más rápido y confiable. Teniendo en cuenta lo an terior, se diseñó y desarrolló un programa de cómputo que permite realizar la selección y evaluación del tren motriz con los componentes comerciales que existen en nuestro país, tanto para un vehículo nuevo como para uno usado; asimismo, cumple con la normatividad que existe en México para limitar velocidad, pesos y dimensiones (SCT, 1994).

El programa de cómputo está basado en la prueba real de desempeño a que puede ser sometido un vehículo, es decir, cumple con la capacidad de arranque y ascenso en pendiente, logrando la operación del motor dentro del régimen óptimo de economía de combustible para reducir el consumo del mismo (Rafael et al., 1995). La evaluación de los factores mencionados permite orientar al transportista sobre los elementos más adecuados para la selección o reconstrucción de los trenes motrices de sus unidades que satisfagan sus necesidades.

\section{Bases para la selección del tren motriz}

Los aspectos principales que se deben tomar en consideración para el proceso de selección de los elementos del tren motriz de un vehículo son:

a) El tipo de actividad: define la naturaleza del transporte -carga o pasajeros- 
y por lo tanto, permite establecer el peso bruto vehicular máximo que puede transportar la unidad.

b) La ruta de operación crítica: este aspecto permite establecer los porcentajes máximos de pendiente ascendente (SCT, 1984), los cuales requerirán potencia adicional para arrancar y superar las pendientes críticas; así también debe considerarse para vencer la resistencia al rodamiento en una carretera en malas condiciones (rugosidad y desgaste de la superficie de la carretera), lo que permite poner particular atención al desgaste de las llantas.

c) El desempeño del vehículo (performance) (Fitch,1993): el desempeño del vehículo se ve afectado principalmente por dos parámetros cuantitativos: el peso de la carga máxima que puede transportar y la pendiente crítica por la que transitará, que a su vez, depende de la ruta de operación. Conociendo estos parámetros se pueden determinar de manera preliminar, la potencia máxima del motor $\mathrm{y}$, por consiguiente, establecer los elementos que integrarán el tipo de vehículo y su tren motriz. Es importante considerar en la selección del tren motriz la economía del combustible, siendo quizás el factor preponderante en la selección de un vehículo para quien debe tomar la decisión en una empresa de transporte.

d) Potencia máxima de un motor: en la selección del tren motriz para mover una carga, la potencia máxima que proporciona un motor - aspecto de gran importancia-, es un criterio insuficiente, ya que no corresponde al mejor desempeño de la unidad, particularmente en la capacidad de arranque en pendientes ( startability) o en la habilidad de ascenso en pendientes (gradeability). (Riley et al., 1990), (SAE, 1997).

e) Cumplimiento de la normatividad para la circulación o construcción de los vehículos en México: este es un elemento clave para la selección del vehículo, ya que se deben observar las normas vigentes en materia de pesos y dimensiones, de protección ecológica (SEMARNAP, 1997) y el acuerdo de velocidad máxima permitida (DDF, 1980); estas normas pueden imponer algunas restricciones de uso, por ejemplo, el combustible Diesel Sin ( de PEMEX), que cumple en México y en los Estados Unidos con las normas ecológicas vigentes respecto a emisiones, pero no a las relativas al ruido de los automotores.

\section{Selección del tren motriz}

La transmisión es el elemento clave para cumplir con características tales como:

- $\quad$ Capacidad de arranque en pendiente ( startability).

- Capacidad de ascenso en pendiente (gradeability).

- Velocidad adecuada de operación.

- Aceleración.

- Capacidad de carga.

Esto debido a que:

1) La velocidad óptima de operación del vehículo permitida por la reglamentación, debe alcanzarse dentro del rango de mínimo consumo específico de com bus ti ble del motor. 
El análisis de patrón de cambios de velocidades [shift pattern] se realiza a través del diagrama de velocidades con el fin de observar el comportamiento de la transmisión; en otras palabras, todos los cambios de engranes de la transmisión se deben realizar dentro del rango de mínimo consumo específico de com bus ti ble del motor.

2) La transmisión influye directamente sobre la capacidad de arranque en pendiente del vehículo. Debido a la relación de paso del primer engrane o marcha, una relación de paso con un valor numéricamente bajo tendrá como consecuencia baja capacidad de arranque, lo cual es importante considerar para el desempeño de la unidad en terreno montañoso.

3) Otra característica asociada con la transmisión, es la capacidad de ascenso del vehículo en pendiente, ya que una transmisión mal seleccionada cuando la unidad se encuentra a su máxima capacidad de carga, puede hacer que el régimen del motor disminuya al grado de no permitir el avance del vehículo.

De lo anterior, se infiere que el elemento que proporciona las características de operación más importantes del vehículo es la transmisión; por esta razón se le considera como base para la selección en el algoritmo desarrollado.

\section{Algoritmo para la selección del tren motriz}

El algoritmo de selección del tren motriz se presenta en el diagrama de flujo de la figura 1.

\section{Entrada de datos}

El primer paso es proporcionar el tipo de vehículo, el peso bruto vehicular y tipo de camino (esto se refiere a la altura sobre el nivel del mar y a la pendiente máxima a transitar). Se obtiene así la primera selección del vehículo que, tomando en cuenta el reglamento de pesos y dimensiones, determina el número de ejes y el número de llantas. El peso bruto vehicular que exceda las disposiciones establecidas en el reglamento es rechazado.

\section{Selección de llantas}

Con la primera selección del vehículo el programa selecciona el tamaño de las llantas, considerando la capacidad de carga de las mismas, su número y el peso bruto ve hicular. El modelo toma en cuenta que cada una de ellas soporta un peso igual al de las restantes, y que sólo estará al $80 \%$ de su capacidad de carga con el fin de prolongar su vida útil (SAE, 1997).

\section{Sección de la transmisión}

La transmisión se selecciona de acuerdo con el peso bruto vehicular de la unidad, considerando el torque nominal de entrada de esta transmisión, la cual se selecciona de una base de datos denominada "transmisión".

\section{Selección del motor}

Para la selección del motor se considera como primera aproximación la potencia necesaria para mover la unidad y para vencer la resistencia aerodinámica del área frontal del vehículo, de acuerdo con la fórmula 1 , 


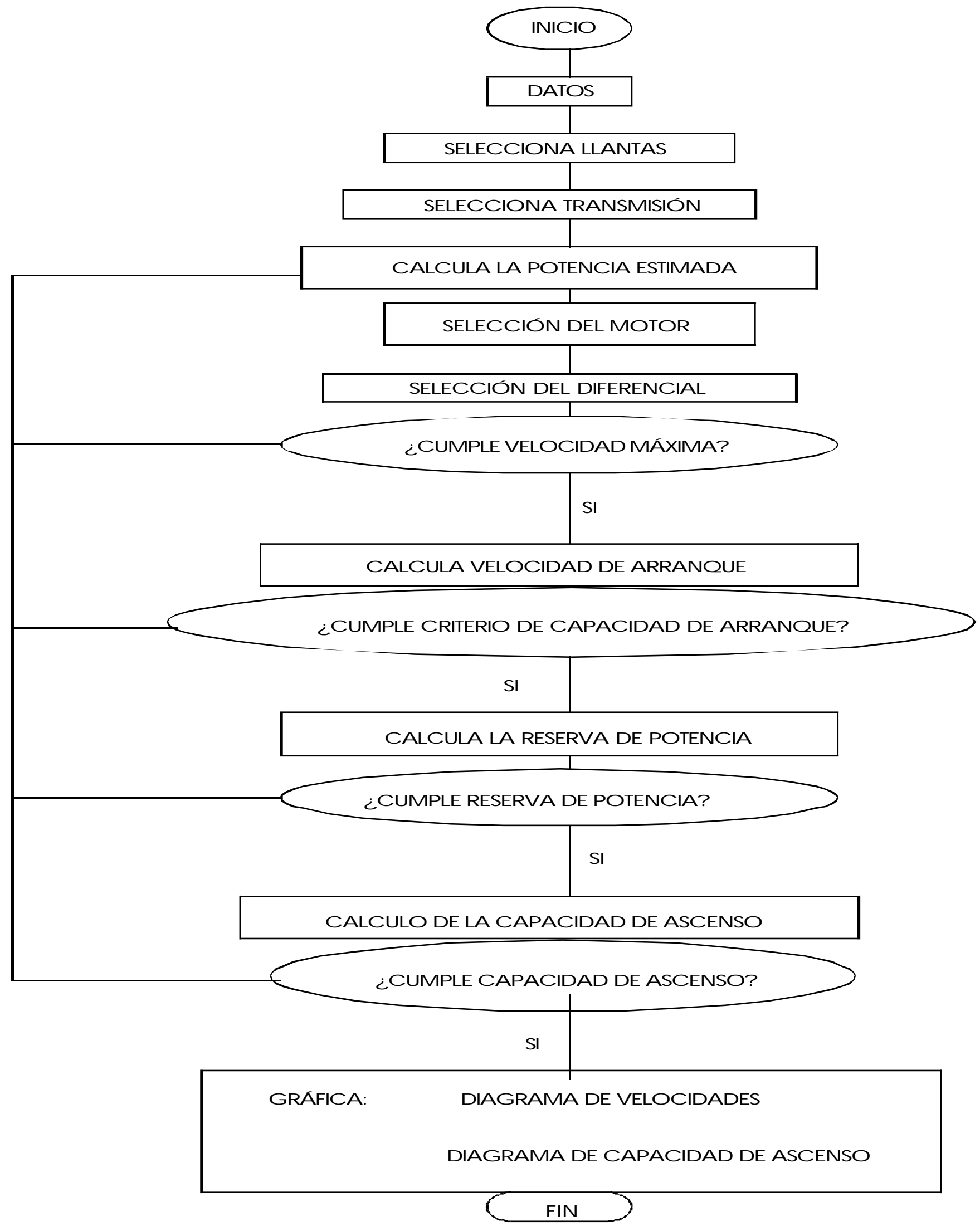

Figura 1 


$$
P e=12.03 S^{*}+2.033 P B V
$$

donde:

$\mathrm{P}_{e}=$ Potencia estimada.

$S$ = Superficie frontal del vehículo.

$P B V=$ Peso Bruto Vehicular.

Esta aproximación, forzará a una primera iteración para encontrar un motor de capacidad adecuada, haciendo que en cada ciclo se incremente la potencia del motor hasta encontrar uno con una potencia que permita mover el peso ve hicu lar de la unidad demasiado grande que implique mayor peso vehicular de la unidad (SCT, 1994). El motor se selecciona de acuerdo a la capacidad torsional de la transmisión y su potencia debe ser mayor a la potencia estimada anteriormente.

\section{Selección del diferencial}

Con la ecuación 2 se calcula el paso del diferencial; el resultado de esta fórmula proporciona una relación de paso cercana a las comerciales y puede utilizarse para alcanzar la máxima velocidad permitida dentro del área de mínimo consumo específico de combustible.

$$
P d=\frac{60 C_{l l}\left(R_{c m}+200\right)}{1000 P_{u} V_{r}}
$$

donde:

$P_{d} \quad=$ Relación de paso del diferencial.

$C_{\| l} \quad=$ Circunferencia de la llanta.

$R_{C m}=$ Régimen de consumo mínimo de combustible delmotor.

$P_{u}=$ Relación de paso del último engrane de la transmisión.
$V_{r}=$ Velocidad reglamentaria de circulación.

Cuando se cumple el criterio de velocidad máxima legal dentro del rango de mínimo consumo de combustible del motor seleccionado, se procede a comprobarlo por medio del despeje de $V_{r}$ de la ecuación 2. El límite de velocidad para un autobús es de 95 $\mathrm{km} / \mathrm{h}$ y para un transporte de carga de 80 $\mathrm{km} / \mathrm{h}$; en ambos casos existe una tolerancia de más $15 \mathrm{~km} / \mathrm{h}$ para maniobras de rebase (Berg, 1996), (Rafael et al., 1995).

\section{Calculo de la capacidad de arranque}

Posteriormente, se evalúa la capacidad de arranque del vehículo de acuerdo con la ecuación 3. La capacidad de arranque se expresa en porcentaje y el óptimo se considera del $25 \%$ cuando el motor está a torque máximo, debido a las condiciones geográficas del país; en caso de no cumplir con este criterio se selecciona una nueva transmisiòn para cumplirlo y se inicia otro ciclo.

$$
S=\frac{T P d P_{1} R_{l l}}{10.7 P V B}
$$

donde:

$S \quad=$ Capacidad de arranque.

$T=$ Torque máximo del motor.

$P_{d} \quad=$ Relación de paso del diferencial.

$P_{1}=$ Relación de paso de la primera velocidad.

$R_{\| l} \quad=$ Revoluciones por kilómetro de la Ilanta.

$P B V=$ Peso bruto vehicular.

Al cumplirse el criterio de capacidad de arranque, se procede a evaluar la potencia 
de reserva del motor de acuerdo a la norma SAE J688; si la reserva es igual a cero, se iniciará otro ciclo de cálculo.

\section{Cálculo de la capacidad de ascenso}

La habilidad de ascenso del vehículo se calcula con la ecuación (4),

$$
G=\frac{37.5 \mathrm{Pr}}{P B V \times 10^{-3} V a}
$$

donde:

$G=\quad$ Habilidad en ascenso en pendiente

$\mathrm{Pr}=$ Potencia de reserva

PBV = Pesobrutovehicular

$\mathrm{Va}=$ Velocidad aparente

El criterio de capacidad de ascenso se fija en $30 \%$, de acuerdo con la información proporcionada por fabricantes de motores (SCT, 1984); en caso de no cumplir con este criterio, se selecciona un motor de mayor potencia para iniciar otra iteración.

Finalmente, se grafican los resultados con el propósito de facilitar su interpretación. Las gráficas consisten en el diagrama de velocidades y la curva de capacidad de ascenso. Se enlistan también las características del vehículo y del tren motriz seleccionado.

En la figura 2, se muestra a manera de ejemplo el patrón de cambio de velocidades cuando se realiza dentro de la zona de mínimo consumo específico de combustible de un vehículo con la siguiente configuración del tren motriz para un tractocamión con $36320 \mathrm{~kg}$ de PBV: motor Cummins NTC 400, transmisión TSP 1414 A, diferencial Eaton R. de P. 5.29, y llantas Goodyear 11.00 R22. Es de notarse que la transmisión no es la más adecuada para un manejo económico de combustible, ya que no todos los cambios caen dentro de régimen de consumo mínimo del motor.

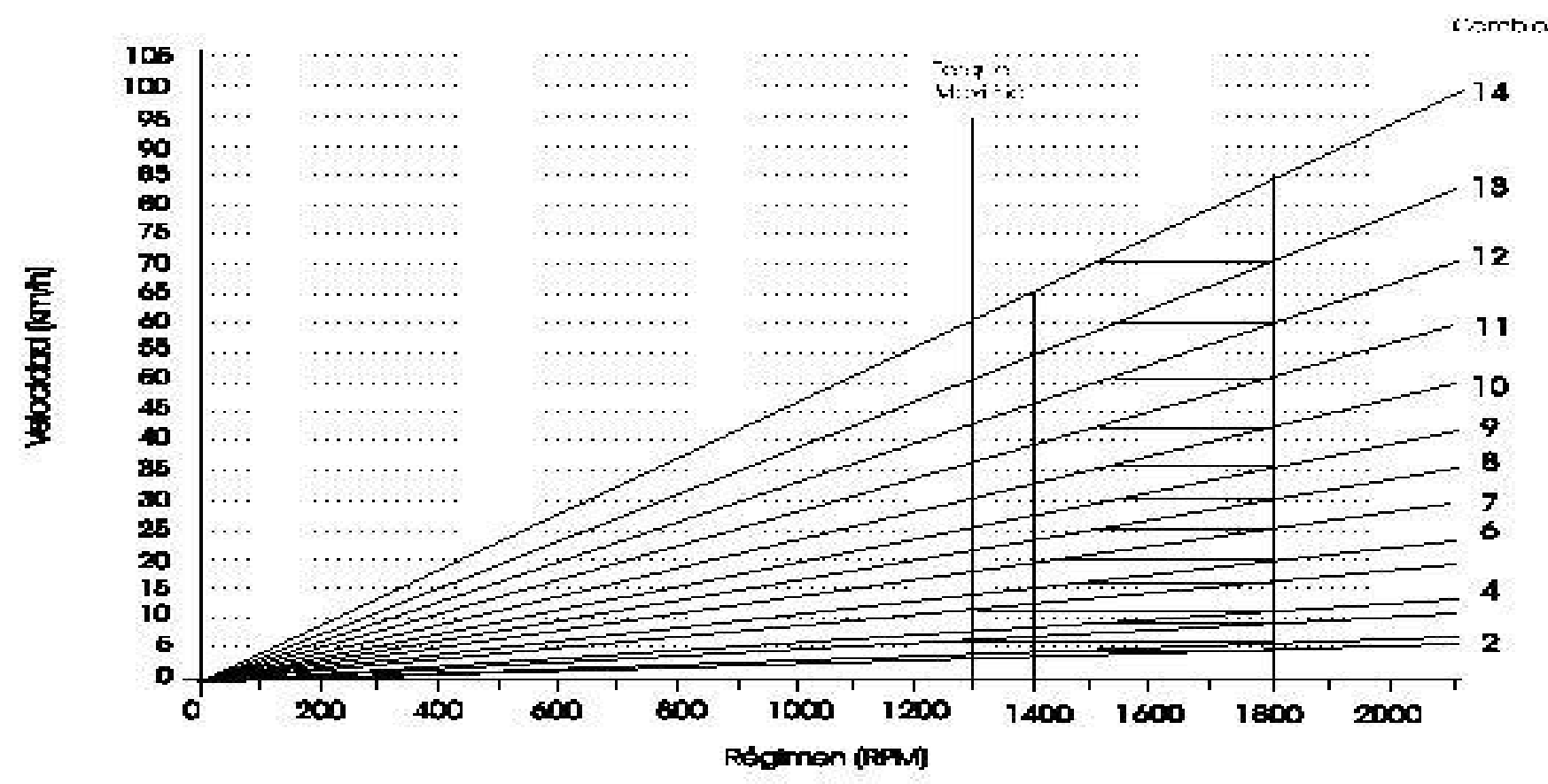

Figura 2. Diagrama de velocidades con el patrón de cambios de velocidad en el régimen de mínimo consumo de combus tible del motor 
En la figura 3 se muestra la gráfica de capacidad de ascenso del mismo vehículo y en ella se observa como disminuye la capacidad de ascenso conforme aumenta la velocidad del vehículo para el mismo tren motriz.

\section{Conclusiones}

Una selección técnica de los vehículos y en particular del tren motriz, conlleva a un conjunto de beneficios significativos para cualquier empresa de autotransporte, sobre todo si se pretenden adquirir diversas unidades. Además de las economías potenciales que se pueden alcanzar en materia de ahorro de energía, el margen de utilidades al que contribuye es importante, porque permite financiar la adquisición de nuevas unidades para renovar o ampliar el parque vehicular y así expandir las operaciones de la empresa. Sin embargo, el conjunto de estos beneficios sólo se pueden lograr cuando la empresa practica una política sistemática de selección vehicular, sustentada técnicamente con el apoyo de herramientas sencillas en el proceso.

El programa diseñado en el Instituto Mexicano del Transporte permite seleccionar el tren motriz para un vehículo nuevo, considerando los aspectos legales y técnicos que afectan la selección de vehículos; asimismo, facilitan la tarea de selección y reducen el tiempo invertido en ella.

El programa permite al transportista realizar modificaciones a las bases de datos de acuerdo con sus necesidades, evaluar el desempeño de un tren motriz existente en sus unidades y auxiliar en la reconstrucción de trenes motrices, si así se requiere.

\section{Referencias}

Berg G. (1996). The Used truck Market, Price is Right. Heavy Duty Trucking. CCE (1993). Guía metodológica de diagnósticos energéticos en el transporte. Comisión de Comunidades Euro-

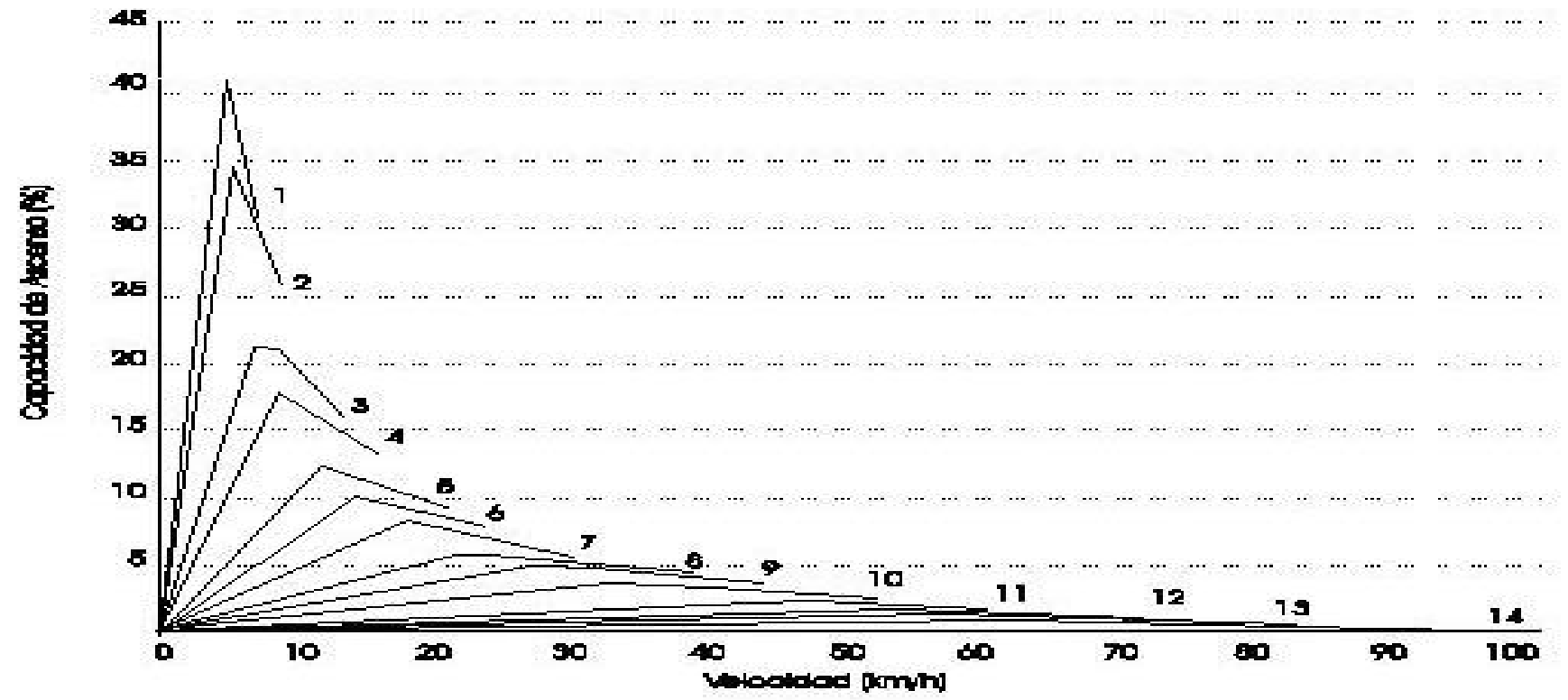

Figura 3. Diagrama de capacidad de ascenso para el vehículo con un tren motriz de: motor Cummins NTC 400; transmisión TSP 1414 A; diferencial Eaton paso 5.29; y llantas Good year 11.00 R22 
peas, Sociedad Francesa de Ingeniería, Cámara Nacional de Empresas de Consultoría y Comisión Nacional para el Ahorro de Energía. México, DF.

DDF (1980). Acuerdo que establece el control de velocidad propulsados por motor a diesel, destinados al servicio de autotransporte de personas y bienes, México, DF. 28 de julio de 1980.

Fitch J.W. (1993). Motor Truck Engineering Handbook. Society of Automotive Engi neers, Warrendale.

Rafael M.M., Manuel F.S. y Guzmán R.C. (1995). Manual de conducción técnica de vehículos automotores diesel. Publicación Técnica No. 70 Instituto Mexicano del Transporte.

Riley T. N., Samuelson E.A. y Schmidt E.A. (1990). New Ten Speed Transmissions Add Unique Features. SAE Technical Papers Series, 902277, Trans mis sion Div.

SAE (1997). Society of Automotive Engineers. Truck Ability Predic tion Proce dure SAE J688, Warrendale.

SCT (1994). Secretaría de Comunicaciones

y Transportes, Norma Oficial Mexicana NOM-012-SCT-2-1994. Sobre el peso y dimensiones máximas con los que pueden circular los vehículos de autotransporte que transitan en los caminos y puentes de jurisdicción federal, México, DF., Diario Oficial de la Federación del 29 de noviembre de 1994.

SCT (1984). Secretaría de Comunicaciones y Transportes, Normas de Servicios Técnicos. Proyectos Geométricos de Carreteras, México, DF.

SEMARNAP (1997). Secretaría del Medio Ambiente, Recursos Naturales y Pesca, Norma Oficial Mexicana NOM-045ECOL-1996. Establece los niveles máximos permisibles de opacidad del humo proveniente del escape de vehículos automotores en circulación que usan diesel o mezclas que incluyan diesel como combustible, México, DF., Diario Oficial de la Federación del 22 de abril de 1997.

\section{Bibliografia sugerida}

CUMMINS (1998). Curvas de eficiencia automotriz. Cummins Engine Company, Inc. Columbus.

EATON (1996). Axles and Brakes Condensed Specifications. Eaton Corporation, Kalamazoo.

GOODYEAR (1997). Factores que afectan la duración de las llantas para un camión. Catálogo Good year, México.

SPICER (1998). Funcionamiento básico de transmisiones y embragues. Transmisiones TSP SPICER, Pedro Escobedo, Qro. 
La selección del tren motriz basada en la eficiencia energética para vehículos de servicio pesado

\section{Semblanza de los autores}

Mercedes Yolanda Rafael-Morales. Es ingeniera industrial en química (Instituto Tecnológico de Celaya, 1978) y maestra en ingeniería química (UNAM, 1989). De 1979 a 1992, fue profesora y jefa del Departamento de Ingeniería Mecánica en el Instituto Tecnológico de Celaya, coordinando la carrera de ingeniería industrial mecánica y la maestría en ingeniería mecánica en diseño. Desde 1993 se desempeña como investigadora, encargada del área de ahorro de energía y emisiones en el Instituto Mexicano del Transporte. Es instructora en los diplomados de ahorro de energía en empresas de transporte, UPIICSA (IPN)-Universidad de Guadalajara; y catedrática en la maestría de ingeniería de tránsito y transporte de la Benemérita Universidad Autónoma de Puebla. Ha impartido 46 cursos de capacitación en la metodología de la conducción técnica para instructores de operadores del transporte público federal. Es asesora en ahorro de energía y protección del ambiente del Sector Transporte del Gobierno Federal y miembro del Grupo de Indicadores de Energía y Medio Ambiente en el Transporte de América del Nortepara el TLC.

Jaime Cervantes de Gortari. Es ingeniero mecánico electricista (UNAM, 1970); maestro en ingeniería mecánica (UNAM, 1972); y doctor (Ph.D.) en ingeniería mecánica (Purdue University, 1976). Es autor y coautor de más de 120 publicaciones (libros de texto, apuntes, monografías de divulgación y artículos originales de investigación). Ha dictado numerosos cursos y conferencias en instituciones nacionales y extranjeras; $y$ ha sido asesor en estudios sobre investigación y educación en ingeniería, así como en proyectos relacionados con el aprovechamiento energético en plantas industriales. Es miembro de honor de la Academia de Ingeniería y miembro regular de la Academia Mexicana de Ciencias. Actualmente es profesor de tiempo completo de la Facultad de Ingeniería de la UNAM y miembro del Sistema Nacional de Investigadores. En el año 2002, la UNAM le otorgó el Premio Universidad Nacional, Docencia en Ciencias Exactas. 